\title{
New methods for obtaining new families of congruent numbers
}

\author{
Hamid Reza Abdolmalki ${ }^{1}$ and Farzali Izadi ${ }^{2}$ \\ ${ }^{1}$ Department of Mathematics, Faculty of Science \\ Azarbaijan Shahid Madani University \\ Tabriz 53751-71379, Iran \\ e-mail: hamid.abdolmalki@gmail.com \\ ${ }^{2}$ Department of Mathematics, Faculty of Science \\ Azarbaijan Shahid Madani University \\ Tabriz 53751-71379, Iran \\ e-mail: f.izadi@urmia.ac.ir
}

Received: 24 August 2018 Revised: 1 February $2019 \quad$ Accepted: 22 February 2019

\begin{abstract}
In this note, we introduce elementary methods for obtaining new families of congruent numbers (CNs). By our methods, we can produce other CNs when one or two CNs are given. Also, we use some of the Pell equations (PEs) for getting some families of CNs. Up to now, it is not exactly determined which prime numbers of the form $p=8 k+1$ are CNs. Among other things, we also introduce two simple methods to find some CNs of the forms $p \equiv 1(\bmod 8)$ and $2 p$ where $p$ is a prime number. By non-CNs and our methods, we also obtain some Diophantine equations (especially of degree 4 ), which have no positive solutions. In the end, we obtain a result on Heron triangles.
\end{abstract}

Keywords: Congruent numbers, Elliptic curves, Rank, Pythagorean triples, Pell equations, Heron triangles.

2010 Mathematics Subject Classification: 11G05, 14H52, 14G05, 11E16, 11D09.

\section{Introduction}

The rational number $n$ is a congruent number $(\mathrm{CN})$ if there are positive rational numbers $a, b, c$ such that $a^{2}+b^{2}=c^{2}$ and $\frac{1}{2} a b=n$ (Otherwise, it is called a non-congruent number.), equivalently, 
there is a Pythagorean triangle (PT) with rational sides and the area equal to $n$. A famous problem regarding $\mathrm{CNs}$ is which rational numbers occur as the area of rational right triangles. This easy looking problem has not been solved completely. It suffices that we study square-free CNs, because from the $\mathrm{CN} \frac{a b}{2}\left(a^{2}+b^{2}=c^{2}\right)$, we conclude that $\frac{k^{2} a b}{2} \equiv \frac{a b}{2}\left((k a)^{2}+(k b)^{2}=(k c)^{2}\right)$ is a $\mathrm{CN}$ and vice versa. Also, note that the notation $\equiv$ means that we omit the square number of the $\mathrm{CN}$.

An elliptic curve over the rationals is a curve $E$ of genus 1 , defined over $\mathbb{Q}$, together with a $\mathbb{Q}$-rational point, and is expressed by the generalized Weierstrass equation of the form

$$
E: y^{2}+a_{1} x y+a_{3} y=x^{3}+a_{2} x^{2}+a_{4} x+a_{6},
$$

where $a_{1}, a_{2}, a_{3}, a_{4}, a_{6} \in \mathbb{Q}$.

A theorem of Mordell-Weil [27] states that the rational points on $E$, form a finitely generated Abelian group $E(\mathbb{Q})$ under a natural group law, i.e., $E(\mathbb{Q}) \cong \mathbb{Z}^{r}(E) \times E(\mathbb{Q})_{\text {tors }}$, where $r(E)$ is a nonnegative integer called the rank of $E$, and $E(\mathbb{Q})_{\text {tors }}$ is the subgroup of elements of finite order in $E(\mathbb{Q})$, called the torsion subgroup of $E(\mathbb{Q})$. The rank of $E$ is the rank of the free part of this group. For more information about elliptic curves, see [27].

In a modern language, $n$ is a $\mathrm{CN}$ if and only if the elliptic curve $E: y^{2}=x^{3}-n^{2} x$ contains a rational point with $y \neq 0$, equivalently, a rational point of infinite order, i.e., the rank of $E$ is non-zero (see [27]). The rank of an elliptic curve is a measure of the size of the set of rational points. However, the question is how one can compute the exact size of this set of rational points. There is no known guaranteed algorithm to determine the rank and it is not known which numbers can occur as the rank of an elliptic curve (see [21]).

The CNs problem has been studied by some authors (see [2-5, 12,13,16-18]) As an example, each of these families has been shown to be CNs:

- $n=2 p_{3}$, i.e., $p_{3} \equiv 3(\bmod 4)$ [Heegner (1952) [15] and Birch (1968) [7]],

- $n=p_{5}, p_{7}$, i.e., $p_{i} \equiv i(\bmod 8)$ [Stephen (1975) [25]],

- $n=p^{u} q^{v} \equiv 5,6,7(\bmod 8), 0 \leq u, v \leq 1$ [B. Groos (1985) [14]],

- $n=2 p_{3} p_{5}, 2 p_{5} p_{7}$,

- $n=2 p_{1} p_{7},\left(\frac{p_{1}}{p_{7}}\right)=1$ [P. Monsky (1990) [20]],

- $n=2 p_{1} p_{3},\left(\frac{p_{1}}{p_{3}}\right)=1$,

where $\left(\frac{p}{q}\right)$ is the Legendre symbol.

A rational triangle is a triangle with rational sides and rational area. A Heron triangle is a triangle with integer sides and integer area. The area $\triangle$ of a triangle having sides $a, b, c$ is given by the well-known formula of Heron $\triangle=\sqrt{s(s-a)(s-b)(s-c)}$, where $s=\frac{(a+b+c)}{2}$ is the semiperimeter $(\mathrm{sP})$ of the triangle. Brahmagupta gave a parametrization for all Heron triangles with sides proportional to

$$
(v+w)\left(u^{2}-v w\right), \quad v\left(u^{2}+w^{2}\right), \quad w\left(u^{2}+v^{2}\right),
$$


where the $\mathrm{sP}$ is equal to $u^{2}(v+w)$, and the area is equal to $u v w(v+w)\left(u^{2}-v w\right)$. There are several open problems concerning the existence of Heron triangles with certain properties (see [9, 24, 28, 29]).

Izadi in [16] obtained some CNs in terms of polynomials by using PTs. For more references about CNs also consult [16]. In this paper, we obtain some new families of CNs in terms of polynomials by elementary methods. In particular, we introduce two methods that find some CNs of the forms $p \equiv 1(\bmod 8)$ and $2 p$ where $p$ is a prime number. Also, by our methods, we may again get some of the results and families of CNs given in [16] and obtain some Diophantine equations (especially of degree 4) which have no positive solutions. We also obtain a result on Heron triangles.

\section{Our results}

\subsection{Producing other $\mathrm{CNs}$ when a $\mathrm{CN}$ is given}

By a simple idea, we obtain some new families of CNs. This method shows how we can produce other $\mathrm{CNs}$ when a $\mathrm{CN}$ is given.

Let $a^{2}+b^{2}=c^{2}$, be a PT where $a, b, c \in \mathbb{N}$. We know that the number $\frac{a b}{2}$ is $\mathrm{CN}$. We try to find other PTs in the form

$$
\left(a x_{1}+b y_{1}+c z_{1}\right)^{2}+\left(a x_{2}+b y_{2}+c z_{2}\right)^{2}=\left(a x_{3}+b y_{3}+c z_{3}\right)^{2},
$$

where $x_{i}, y_{i}, z_{i} \in \mathbb{N}$ are variables. Then, the number $\frac{\left(a x_{1}+b y_{1}+c z_{1}\right)\left(a x_{2}+b y_{2}+c z_{2}\right)}{2}$ will be a $\mathrm{CN}$. After some simplifications, (1) becomes

$$
\begin{gathered}
\left(x_{1}^{2}+x_{2}^{2}\right) a^{2}+\left(y_{1}^{2}+y_{2}^{2}\right) b^{2}+\left(z_{1}^{2}+z_{2}^{2}\right) c^{2}+2 a b\left(x_{1} y_{1}+x_{2} y_{2}\right)+2 a c\left(x_{1} z_{1}+x_{2} z_{2}\right)+2 b c\left(y_{1} z_{1}+y_{2} z_{2}\right)= \\
x_{3}^{2} a^{2}+y_{3}^{2} b^{2}+z_{3}^{2} c^{2}+2 a b x_{3} y_{3}+2 a c x_{3} z_{3}+2 b c y_{3} z_{3} .
\end{gathered}
$$

Then, by using the relation $a^{2}+b^{2}=c^{2}$, we can take

$$
\begin{aligned}
x_{1}^{2}+z_{1}^{2}+x_{2}^{2}+z_{2}^{2} & =x_{3}^{2}+z_{3}^{2}, \\
x_{1} z_{1}+x_{2} z_{2} & =x_{3} z_{3}, \\
y_{1}^{2}+z_{1}^{2}+y_{2}^{2}+z_{2}^{2} & =y_{3}^{2}+z_{3}^{2}, \\
y_{1} z_{1}+y_{2} z_{2} & =y_{3} z_{3}, \\
x_{1} y_{1}+x_{2} y_{2} & =x_{3} y_{3} .
\end{aligned}
$$

Then, any solution for the above Diophantine system will produce a CN. As an example, by letting $x_{1}=y_{2}, x_{2}=y_{1}, x_{3}=y_{3}$ in the system, we will have $z_{1}=z_{2}, x_{1} \neq x_{2}$ and the system becomes

$$
\begin{aligned}
x_{1}^{2}+x_{2}^{2}+2 z_{1}^{2} & =x_{3}^{2}+z_{3}^{2}, \\
\left(x_{1}+x_{2}\right) z_{1} & =x_{3} z_{3}, \\
2 x_{1} x_{2} & =x_{3}^{2} .
\end{aligned}
$$


By squaring the second relation, replacing $2 x_{1} x_{2}=x_{3}^{2}$, and putting $z_{1}^{2}=2 x_{1} x_{2}, z_{3}=x_{1}+x_{2}$, the first relation is established. As an example, by letting $x_{1}=y_{2}=1, x_{2}=y_{1}=2, x_{3}=y_{3}=$ $z_{1}=z_{2}=2, z_{3}=3$ we obtain

$$
(a+2 b+2 c)^{2}+(2 a+b+2 c)^{2}=(2 a+2 b+3 c)^{2} .
$$

By letting $x_{1}=y_{2}=2, x_{2}=y_{1}=9, x_{3}=y_{3}=z_{1}=z_{2}=6, z_{3}=11$ we get $(2 a+9 b+$ $6 c)^{2}+(9 a+2 b+6 c)^{2}=(6 a+6 b+11 c)^{2}$.

Finally, we conclude that $\frac{(a+2 b+2 c)(2 a+b+2 c)}{2}$ and $\frac{(2 a+9 b+6 c)(9 a+2 b+6 c)}{2}$ are CNs. Then, by letting $a=t^{2}-s^{2}, b=2 t s, c=t^{2}+s^{2}$, so that the relation $a^{2}+b^{2}=c^{2}$ holds, we get two families of CNs. As an example, by letting $a=3, b=4, c=5$ in the above identities we get $20^{2}+21^{2}=29^{2}$, and $65^{2}+72^{2}=97^{2}$, then conclude that $\frac{20 \cdot 21}{2}=210$, and $\frac{65 \cdot 72}{2} \equiv 65$ are CNs. Note that by replacing $a \rightarrow-a$ or $b \rightarrow-b$ or $c \rightarrow-c$ in the relation $a^{2}+b^{2}=c^{2}$, we can obtain other identities such as:

$$
\begin{aligned}
(-a+2 b+2 c)^{2}+(-2 a+b+2 c)^{2} & =(-2 a+2 b+3 c)^{2}, \\
(a-2 b+2 c)^{2}+(2 a-b+2 c)^{2} & =(2 a-2 b+3 c)^{2}, \\
(a+2 b-2 c)^{2}+(2 a+b-2 c)^{2} & =(2 a+2 b-3 c)^{2}, \\
(a-2 b-2 c)^{2}+(2 a-b-2 c)^{2} & =(2 a-2 b-3 c)^{2} .
\end{aligned}
$$

Then, each of the numbers $\frac{(-a+2 b+2 c)(-2 a+b+2 c)}{2}=s(t+s)(t+2 s)(t+3 s), \frac{(a-2 b+2 c)(2 a-b+2 c)}{2}$, $\frac{(a+2 b-2 c)(2 a+b-2 c)}{2}$, and $\frac{(a-2 b-2 c)(2 a-b-2 c)}{2}$ are CNs. This is correct for all of our cases.

Remark 2.1. By letting $s=k^{2}$ in the $C N \frac{(-a+2 b+2 c)(-2 a+b+2 c)}{2}=s(t+s)(t+2 s)(t+3 s)$, we conclude that there are infinitely many $C N$ s in the form $\left(t+k^{2}\right)\left(t+2 k^{2}\right)\left(t+3 k^{2}\right)$, which is the product of three consecutive terms of an arithmetic progression. If we set $t=g^{2}-k^{2}$, we get the $C N g^{2}\left(g^{2}+k^{2}\right)\left(g^{2}+2 k^{2}\right) \equiv\left(g^{2}+k^{2}\right)\left(g^{2}+2 k^{2}\right)$.

Similarly, we conclude that if $a^{2}+b^{2}=c^{2}$, then we have

$$
\left(2 p^{2} a+q^{2} b+2 p q c\right)^{2}+\left(q^{2} a+2 p^{2} b+2 p q c\right)^{2}=\left(2 p q a+2 p q b+\left(2 p^{2}+q^{2}\right) c\right)^{2},
$$

i.e., for every $t, s, p, q$, the number

$$
\left(p^{2}\left(t^{2}-s^{2}\right)+q^{2} s t+p q\left(t^{2}+s^{2}\right)\right)\left(q^{2}\left(t^{2}-s^{2}\right)+4 p^{2} s t+2 p q\left(t^{2}+s^{2}\right)\right)
$$

is a CN. If $p=q=t=1$, then we conclude that $(s+1)(s+2)(s+3)$ is a CN. This shows that the product of three consecutive integers is a $\mathrm{CN}$, which is mentioned in [16], too. If we set $s+3=k^{2}$, we conclude that $(s+1)(s+2)(s+3) \equiv\left(k^{2}-2\right)\left(k^{2}-1\right)$ is a $\mathrm{CN}(k \neq 1 \in \mathbb{N})$ which is in the form of product of two consecutive integers. If $s=1$ and $t=2$, then we see that $(q+p)(q+2 p)(2 q+3 p)$ is a $\mathrm{CN}(p, q \in \mathbb{N})$. Letting $s+2=k^{2}$ leads to $k^{4}-1$ is a $\mathrm{CN}$, which is also mentioned in [16]. As an example, if $k=3$, we conclude that $80 \equiv 5$ is a CN. 


\subsection{CNs of the forms $p \equiv 1(\bmod 8)$ and $2 p$ where $p$ is a prime number}

We introduce an easily checked method which finds some of CNs of the form $p=8 k+1$ ( $p$ is a prime number.). Up to now, it is not exactly determined which prime numbers of the form $p=8 k+1$ are $\mathrm{CNs}$ (see [16]). For instance, 17 is known to be non-congruent, while 41 is known to be congruent.

We assert that every prime number of the form $P=8 k+1=a^{2}+b^{2}$ (Note that every prime number of the form $P=4 k+1$ is uniquely written in the form of $P=a^{2}+b^{2}$.) in which $a^{2}-b^{2}:=u^{2}$ is a square integer (This work can be checked by an easy computer search.), is a $\mathrm{CN}$. This result is followed by using the congruent number $s t\left(s^{2}-t^{2}\right)=\operatorname{st}(s+t)(s-t)$ and letting $s=a^{2}, t=b^{2}, P=a^{2}+b^{2}$, and $s-t=a^{2}-b^{2}=u^{2}$ in the above family. Then, the number $s t(s+t)(s-t)=a^{2} b^{2}\left(a^{2}+b^{2}\right)\left(u^{2}\right) \equiv a^{2}+b^{2}=P$ will be $\mathrm{CN}$. As two examples, since we have $41=4^{2}+5^{2}, 5^{2}-4^{2}=3^{2}$, and $353=8^{2}+17^{2}, 17^{2}-8^{2}=15^{2}$, then the prime numbers 41 and 353 are CNs.

To get other examples, let $p=n^{2}+(n-1)^{2}$ and $n^{2}-(n-1)^{2}=c^{2}$. Then, we get $p=2 n^{2}-2 n+1$ and $n=\frac{c^{2}+1}{2}$. Now by using the above idea and letting $c=3,5,7, \ldots$, such that $p$ is prime, we obtain the CNs $p=4^{2}+5^{2}, 12^{2}+13^{2}, 24^{2}+25^{2}, 60^{2}+61^{2}, 84^{2}+85^{2}$, $144^{2}+145^{2}, \ldots$, which are also prime numbers of the form $p=8 k+1$.

Note that the above idea is correct for any composite number, i.e., $c^{2}-b^{2}:=a^{2}\left(a^{2}+b^{2}=c^{2}\right)$ yields that the numbers $b^{2}+c^{2}$ and $a^{2}+c^{2}$ (due to symmetry) are CNs. Thus, by using the CN $\frac{a b}{2}\left(a^{2}+b^{2}=c^{2}\right)$, we can produce the CNs $b^{2}+c^{2}$ and $a^{2}+c^{2}$. Now we obtain all of the results of Corollary 3.2 given in [16] by a different easy method, again. By letting $s=a^{2}, t=b^{2}$, in the CN $s t\left(s^{2}-t^{2}\right)=s t(s+t)(s-t)=a^{2} b^{2}\left(a^{2}+b^{2}\right)\left(a^{2}-b^{2}\right)$ and setting $a^{2}+b^{2}=c^{2}$, we get the $\mathrm{CN} a^{2}-b^{2}$, i.e., $a^{2}+b^{2}=c^{2}$ yields that $a^{2}-b^{2}$ is a CN. Letting $s=c$ and $t=b$ in the $\mathrm{CN} s t\left(s^{2}-t^{2}\right)=b c\left(c^{2}-b^{2}\right)$ and setting $c^{2}-b^{2}=a^{2}$, yields the $\mathrm{CN} b c$, i.e., $a^{2}+b^{2}=c^{2}$ leads to the CNs $b c$ and $a c$ (similarly). Then, from $a^{2}+b^{2}=c^{2}$, we conclude that all of the numbers $a c, b c,\left|a^{2}-b^{2}\right|, a^{2}+c^{2}$, and $b^{2}+c^{2}$ are CNs. This is one of the main results given in [16]. So, we can obtain some of the other main results given in [16] by these methods, again.

Example 2.2. By using the PT $(3,4,5)$ (with corresponding $C N 6$ ), we get the square-free $C N s$ $15,20 \equiv 4,7,34,41$.

\subsection{CNs and PEs}

Pell's equation is a Diophantine equation of the form $x^{2}-d y^{2}=1, x, y \in \mathbb{Z}$, where $d$ is a given natural number which is not a square. An equation of the form $x^{2}-d y^{2}=a$ for an integer $a$ is usually referred to as a Pell-type equation. For more information about Pell's equation, the reader can see any textbook on number theory.

Now, we study the PE $x^{2}-p y^{2}=-1$ where $p$ is a prime number and relate it to CNs. It is clear that if this equation has a solution, then $p \equiv 1(\bmod 4)$ because we have $x^{2} \equiv-1(\bmod p)$. It is well-known that the general PE $x^{2}-d y^{2}=1$ has infinitely many positive integer solutions when $d$ is a given non-square positive integer, but it is possible that there exists no solution of the 
PE $x^{2}-d y^{2}=-N^{2}$. For more information about PE, the reader may consult $[1,22]$. First we mention the following theorem, which can be found in every book on number theory:

Theorem 2.3. The PE $x^{2}-p y^{2}=-1$, where $p \equiv 1(\bmod 4)$ is an arbitrary prime number, has infinitely many solutions in natural numbers.

Now we introduce our main result. We know that $\left(x^{2}-1,2 x, x^{2}+1\right)$ is a PT and the number $2 x\left(x^{2}+1\right)$ is a $\mathrm{CN}$. Then, by replacing $x^{2}+1$ by $p y^{2}$ in the $\mathrm{CN} 2 x\left(x^{2}+1\right)$ (Note that we are going to relate the CN $2 x\left(x^{2}+1\right)$ to the PE $x^{2}-p y^{2}=-1$.), we conclude that in the PE $x^{2}-p y^{2}=-1$, the number $2 x\left(x^{2}+1\right)=2 x p y^{2} \equiv 2 x p$ is a $\mathrm{CN}$. In particular, if $2 x$ (or $x$ ) is a square number, then $p$ (or $2 p$ ) will be a CN. Then, we must find the solutions of this equation such that $2 x$ (or $x$ ) is a square number which is possible by an easy computer search. It is interesting that this method finds some of CNs of the forms $p$ and $2 p$, in which $p$ is prime of the form $8 k+1$. As an example, the solution $(18,5)$ of the PE equation $x^{2}-13 y^{2}=-1$, finds the CN $2 \cdot 18 \cdot 13 \equiv 13$. The solutions $(4,1)$ and $(268,65)$ of the PE $x^{2}-17 y^{2}=-1$, discovers the CNs $2 \cdot 4 \cdot 17 \equiv 34$ and $2 \cdot 268 \cdot 17 \equiv 2 \cdot 17 \cdot 67$.

\subsection{CNs and some DEs which have no solutions in $\mathbb{N}$}

In this part, by the PEs, we obtain a family of CNs in which any term is the product of some prime numbers. From part 1, we know that the number $s(s-1)(s+1)=s\left(s^{2}-1\right)$ is a $\mathrm{CN}$ $(s>1 \in \mathbb{N})$. Let $s^{2}-1=d y^{2}$, where $d$ is a non-square positive integer. Then, the number $s\left(s^{2}-1\right) \equiv s d$ will be a CN. We have $s^{2}-d y^{2}=1$. Then, we need to obtain the solutions of the PE $x^{2}-d y^{2}=1$ and so the number $x d$ will be a CN. As an example, we know that all of the positive solutions of the PE $x^{2}-2 y^{2}=1$ are $\left(x_{n}, y_{n}\right)$ where $(3+2 \sqrt{2})^{n}=\left(x_{n}+y_{n} \sqrt{2}\right)$. Also, we have $(x, y)=\left(\frac{Q_{2 n}}{2}, P_{2 n}\right)$ where $Q_{n}=\alpha^{n}+\beta^{n}, P_{n}=\frac{\alpha^{n}-\beta^{n}}{\alpha-\beta}, \alpha=1+\sqrt{2}$ and $\beta=1-\sqrt{2}$. Then, any term of the set of $\left\{Q_{2 n}: n \in \mathbb{N}\right\}$ is a CN. As an example, $2 x_{1}=2 \cdot 3=6$, $2 x_{2}=2 \cdot 17=34,2 x_{3}=2 \cdot 99 \equiv 22$ are CNs. By using the solutions of $x^{2}-3 y^{2}=1$, we conclude that $3 x_{4}=3 \cdot 97=291$ is a CN. Similarly, by using the general PE $x^{2}-d y^{2}=N^{2}(d$ is a non-square positive integer) and replacing $x^{2}-N^{2}$ by $d y^{2}$ in the $\mathrm{CN} x N\left(x^{2}-N^{2}\right)$, we get the $\mathrm{CN} x N\left(x^{2}-N^{2}\right) \equiv x N d$, i.e., in the PE $x^{2}-d y^{2}=N^{2}$, the number $x N d$ is a CN. Also, using the solutions of the PE $x^{2}-d y^{2}=-N^{2}$ (If there exists any solution of it.) and replacing $x^{2}+N^{2}$ by $d y^{2}$ in the $\mathrm{CN} 2 x N\left(x^{2}+N^{2}\right)$, leads to the $\mathrm{CN} 2 x N d$. As an example, the solutions $(x, y)=(7,5),(41,29)$ of $x^{2}-2 y^{2}=-1$ leads to the CNs $2 \cdot 7 \cdot 1 \cdot 2 \equiv 7$ and $2 \cdot 41 \cdot 1 \cdot 2 \equiv 41$.

It is interesting to see that with the results of the above idea, we can show that some Diophantine equations (DEs) have no solutions in $\mathbb{N}$. We know that in the PE $x^{2}-d y^{2}=N^{2}$ where $d$ is a square-free number, the number $x N\left(d y^{2}\right) \equiv x N d$ is a $\mathrm{CN}$ (note that $x \neq N$, because in the PT $\left(x^{2}-N^{2}, 2 x N, x^{2}+N^{2}\right)$, we have $\left.x \neq N\right)$. Now if $x, N, d$ are chosen so that $x N d$ is a non-CN, then the DE $x^{2}-d y^{2}=N^{2}$ will not have any solutions in $\mathbb{N}$. As an example, in the number $x N d$, let $d$ be a non-CN and $N=N^{\prime 2}$. Now it is clear that if $x$ is a square, say $x^{\prime 2}$, then the number $x N d \equiv x^{\prime 2} N^{\prime 2} d \equiv d$ will be a non-CN (note that $d$ is a non-CN) that is a contradiction because $x N d$ is a $\mathrm{CN}$. This yields that the $\mathrm{DE} x^{\prime 4}-d y^{2}=N^{\prime 4}$ has no solutions in $\mathbb{N}\left(x^{\prime} \neq N^{\prime}\right)$ where $d$ is an arbitrary non-CN which is not a square. As two examples, the DEs $x^{\prime 4}-2 y^{2}=1$ (This 
means that in the PE $x^{2}-2 y^{2}=1$, the variable $x$ cannot be a square.) and $x^{4}-2 y^{4}=N^{\prime 4}$ (or $2 y^{4}+N^{\prime 4}=x^{\prime 4}$ ) have no solutions in $\mathbb{N}$. Euler conjectured that the $\mathrm{DE} A^{4}+B^{4}+C^{4}=D^{4}$, or more generally $A_{1}^{N}+A_{2}^{N}+\cdots+A_{N-1}^{N}=A_{N}^{N},(N \geq 4)$, has no solution in $\mathbb{N}$ (see [10]). Nearly two centuries later, a computer search (see [19]) found the first counterexample to the general conjecture (for $N=5$ ): $27^{5}+84^{5}+110^{5}+133^{5}=144^{5}$.

In 1986, Noam Elkies, by using the elliptic curves theory, found an infinite series of counterexamples for the case $K=4$ (see [11]). His smallest counterexample was:

$$
2682440^{4}+15365639^{4}+18796760^{4}=20615673^{4} .
$$

This shows that the DE $A^{4}+B^{4}+C^{4}=D^{4}$ has infinitely many solutions in $\mathbb{N}$ but the DE $2 y^{4}+N^{\prime 4}=x^{\prime 4}$ has no solutions.

Similarly, let $N d=t^{2}, x=x^{\prime 2}$ (we know that square numbers are not CNs). Then, there exist $k, p, q$ such that we have $N=k p^{2}, d=k q^{2},(p, q)=1$ and $t=k p q$. Thus, the DE $x^{4}+k Y^{2}=k^{2} N^{\prime 4}$, where $k$ is an arbitrary positive integer which is not a square, has no solution in $\mathbb{N}$ (because $d$ is not square). So the quartic DE $x^{\prime 4}+k Y^{4}=k^{2} N^{\prime 4}$ has no solutions with the above condition on $\mathrm{k}$, either.

Similarly in the PE $x^{2}-d y^{2}=-N^{2}$ (if there exist any solutions), the number $2 x N\left(x^{2}+N^{2}\right)=$ $2 x N\left(d y^{2}\right) \equiv 2 x N d$ is a $\mathrm{CN}(x \neq N)$. Then, if $x, N, d$ are chosen such that $2 x N d$ be non-CN, then the DE $x^{2}-d y^{2}=N^{2}$ will not have any solutions in $\mathbb{N}$. As an example, by replacing $x \rightarrow 2 x^{\prime 2}, N \rightarrow N^{\prime 2}$ in the above PE and letting $d$ as a non-CN which is not a square, we conclude that the DE $4 x^{\prime 4}-d y^{2}=N^{\prime 4}$ has no solutions in $\mathbb{N}$. As examples, the DEs $4 x^{\prime 4}=2 y^{4}+N^{\prime 4}$ and $4 x^{\prime 4}=3 y^{4}+N^{\prime 4}$ have no solutions in $\mathbb{N}$ (note that these DEs have no solutions in $\mathbb{Q}$, too, because these DEs are homogenous). By this method, we can obtain other DEs which have no solutions in $\mathbb{N}$.

\subsection{CNs and an identity}

We prove that if $a^{2}+b^{2}=c^{2}+d^{2}$, then the numbers $\frac{(a c \pm b d)(a d \mp b c)}{2}$ will be CNs. We have two identities

$$
(a c \pm b d)^{2}+(a d \mp b c)^{2}=\left(a^{2}+b^{2}\right)\left(c^{2}+d^{2}\right) .
$$

It can be easily shown that all integer solutions to the equation $a^{2}+b^{2}=c^{2}+d^{2}$ are

$$
a=\frac{1}{2}(m n+p q), \quad b=\frac{1}{2}(m p-n q), \quad c=\frac{1}{2}(m p+n q), \quad d=\frac{1}{2}(m n-p q),
$$

where $m, n, p, q$ are integers. Then, for these values of $a, b, c, d$, the numbers $\frac{(a c \pm b d)(a d \mp b c)}{2}$ are CNs.

Also, by letting $a=p+q, b=r-s, c=p-q$ and $d=r+s$ such that $p q=r s$, we will have $a^{2}+b^{2}=c^{2}+d^{2}$. Then, we conclude that if $p q=r s$, so two new families of $\left(p^{2}-q^{2}+r^{2}-s^{2}\right)(p s+q r)$ and $\left(p^{2}-q^{2}-r^{2}+s^{2}\right)(p r+q s)$ will be CNs.

By letting $a^{2}+b^{2}=k r^{2}$ and $c^{2}+d^{2}=k w^{2}$ in the above identity, we get

$$
(a c \pm b d)^{2}+(a d \mp b c)^{2}=\left(a^{2}+b^{2}\right)\left(c^{2}+d^{2}\right)=k r^{2} \dot{k} w^{2}=k^{2} r^{2} w^{2}=(k r w)^{2} .
$$


Then, the numbers $\frac{(a c \pm b d)(a d \mp b c)}{2}$ will be CNs.

If $k=1$ then $(a, b, r)$ and $(c, d, w)$ are PTs. For example, with PTs $(3,4,5)$ and $(5,12,13)$ we get the CNs $n=\frac{(3 \times 12+4 \times 5)(3 \times 5-12 \times 4)}{2} \equiv 231$ and $n=\frac{(3 \times 12-4 \times 5)(3 \times 5+12 \times 4)}{2} \equiv 14$. This method introduces a way for producing new CNs when two CNs are given.

For $k=m^{2}+n^{2}$, let $\{a, b, r\}=\left\{s^{2} m-k t^{2} m, n s^{2}-2 k s t+k n t^{2}, s^{2}-2 n s t+k t^{2}\right\}$ and $\{c, d, w\}=\left\{S^{2} m-k T^{2} m, n S^{2}-2 k S T+k n T^{2}, S^{2}-2 n S T+k T^{2}\right\}$, then $a^{2}+b^{2}=k r^{2}$ and $c^{2}+d^{2}=k w^{2}$. Hence $\frac{(a c \pm b d)(a d \mp b c)}{2}$ will be a CN.

\subsection{Other families of CNs}

In the following, we mention some other families of CNs. We know that if $(a, b, c)$ is a PT, then $\left(2 a c, b^{2}, a^{2}+c^{2}\right)$ is a PT, too. (see [16]) By repeating, we conclude that $\left(4 a c\left(a^{2}+c^{2}\right), b^{4}\right.$, $\left.\left(a^{2}+c^{2}\right)^{2}+(2 a c)^{2}\right)=\left(A^{\prime}, B^{\prime}, C^{\prime}\right)$ is a PT. Then, we get that $2 a c\left(a^{2}+c^{2}\right)$ is a CN. Similarly, we conclude that $2 b c\left(b^{2}+c^{2}\right)$ is a $\mathrm{CN}$. Also, $A^{\prime} C^{\prime}, B^{\prime} C^{\prime}$ are $\mathrm{CNs} \Rightarrow a c\left(a^{2}+c^{2}\right)\left(a^{4}+c^{4}+6 a^{2} c^{2}\right)$, and $b c\left(b^{2}+c^{2}\right)\left(b^{4}+c^{4}+6 b^{2} c^{2}\right)$ are CNs.

Similarly (see [16]), $(a, b, c) \Rightarrow\left(2 b c, a^{2}, b^{2}+c^{2}\right) \Rightarrow\left(2 a^{2}\left(b^{2}+c^{2}\right), 4 b^{2} c^{2}, a^{4}+\left(b^{2}+c^{2}\right)^{2}\right)=$ $\left(A^{\prime}, B^{\prime}, C^{\prime}\right) . B^{\prime} C^{\prime}$ is a $\mathrm{CN} . \Rightarrow a^{4}+b^{4}+c^{4}+2 b^{2} c^{2}=2\left(b^{4}+c^{4}\right)$ and similarly $2\left(a^{4}+c^{4}\right)$ are CNs.

$$
(a, b, c) \Rightarrow\left(2 a b, b^{2}-a^{2}, c^{2}\right) \Rightarrow\left(4 a b\left(b^{2}-a^{2}\right),\left(b^{2}-a^{2}\right)^{2}-4 a^{2} b^{2}, c^{4}\right)=\left(A^{\prime}, B^{\prime}, C^{\prime}\right) .
$$

$A^{\prime} C^{\prime}$ and $B^{\prime} C^{\prime}$ are $\mathrm{CNs} \Rightarrow a b\left(b^{2}-a^{2}\right)$ and $b^{4}+a^{4}-6 a^{2} b^{2}$ are CNs.

Note that, applying the formulas recursively, we can obtain in infinite families of PTs and CNs from any PT.

\subsection{A family of Heron triangles with some properties}

We obtain a family of Heron triangles by an elementry method. In the Heron formula let $s=t_{1}^{2}$, $s-a=t_{2}^{2}, s-b=t_{3}^{2}$ and $s-c=t_{4}^{2}$. Then, we get $a=t_{1}^{2}-t_{2}^{2}, b=t_{1}^{2}-t_{3}^{2}, c=t_{1}^{2}-t_{4}^{2}$.

Also the relation $s=\frac{(a+b+c)}{2}$ yields

$$
t_{1}^{2}=t_{2}^{2}+t_{3}^{2}+t_{4}^{2}
$$

Now it is clear that any solution of (3) produces a Heron triangle with sides $a, b, c$ in terms of $t_{i}$ with the area equal to $\left|t_{1} t_{2} t_{3} t_{4}\right|$. A parametric solution of (3) is given by

$$
t_{1}=l^{2}+m^{2}+n^{2}, \quad t_{2}=l^{2}+m^{2}-n^{2}, \quad t_{3}=2 l n, \quad t_{4}=2 m n,
$$

where $m, n, l \in \mathbb{N}$.

Then, we get a family of Heron triangles as follows

$$
\begin{aligned}
a & =4 n^{2}\left(l^{2}+m^{2}\right), \\
b & =l^{4}+m^{4}+n^{4}+2 l^{2} m^{2}+2 m^{2} n^{2}-2 l^{2} n^{2}, \\
c & =l^{4}+m^{4}+n^{4}+2 l^{2} m^{2}-2 m^{2} n^{2}+2 l^{2} n^{2}, \\
\triangle & =4 l m n^{2}\left|l^{4}+m^{4}-n^{4}+2 l^{2} m^{2}\right|, \\
P & =2\left(l^{2}+m^{2}+n^{2}\right)^{2} .
\end{aligned}
$$


It can be easily checked that the conditions $a+b>c, a+c>b, b+c>a$ on the sides of the triangle imply that we must have $l^{2}+m^{2} \neq n^{2}$. As an example, by letting $l=m=n=1$ and $m=n=2, l=1$, we get the triangles of $(5,5,8)$ and $(17,65,80)$ with the areas equal to 12 and 288 , respectively.

Now we obtain Heron triangles with two equal sides. It is clear that by letting $t_{2}=t_{3}$ in (3), the two sides of the triangle $a, b$ will be equal. Now we have $t_{1}^{2}=2 t_{2}^{2}+t_{4}^{2}$ or $\left(t_{1}-t_{4}\right)\left(t_{1}+t_{4}\right)=2 t_{2}^{2}$. By letting $\left(t_{1}-t_{4}\right)=A$ and $\left(t_{1}+t_{4}\right)=B$ we get $A B=2 t_{2}^{2}$ and conclude that $A$ or $B$ is even. Letting $A=2 A^{\prime}$ yields $t_{2}^{2}=A^{\prime} B$. All of the integer solutions of this equation are $A^{\prime}=k M^{2}$, $B=k N^{2}, t_{2}=t_{3}=k M N$, where $(M, N)=1$. Then, we get $t_{1}=\frac{A+B}{2}=\frac{2 k M^{2}+k N^{2}}{2}$ and $t_{4}=\frac{B-A}{2}=\frac{k N^{2}-2 k M^{2}}{2}$. Finally, by taking $N=2 N^{\prime}$ and removing the factor of $k$, we obtain $t_{1}=M^{2}+2 N^{\prime 2}, t_{2}=t_{3}=2 M N^{\prime}, t_{4}=2 N^{\prime 2}-M^{2}$ and a family of Heron triangles as follows $a=b=t_{1}^{2}-t_{2}^{2}=M^{4}+4 N^{\prime 4}, c=t_{1}^{2}-t_{4}^{2}=8 M^{2} N^{\prime 2}$ whose area and perimeter are $S=t_{1} t_{2}^{2} t_{4}=4 M^{2} N^{\prime 2}\left(4 N^{\prime 4}-M^{4}\right)$ and $P=2\left(M^{2}+2 N^{\prime 2}\right)$. Note that letting $M^{2}=G$ and $2 N^{\prime 2}=F$ yields $a=b=G^{2}+F^{2}, c=4 G F, S=2 G F\left|F^{2}-G^{2}\right|$, and $P=2(G+F)^{2}$. Also, the inequalities of the sides show that we must have $F \neq G$, and $F, G \neq 0$. As two examples by taking $G=1, F=3$ and $G=2, F=3$, we obtain the Heron triangles of $(10,10,12)$ and $(13,13,24)$ with two equal sides and the areas equal to 48,60 , respectively. The above formula shows for every prime number of the form $p=4 t+1$, there exists a Heron triangle with the sides $a=b=p$, because any $p$ in this form is written as $p=G^{2}+F^{2}$.

Now, by using the above family, we obtain infinitely many triples of Heron triangles with equal areas such that each triangle has two equal sides. To equate the areas, we must solve the Diophantine equation

$$
G_{1} F_{1}\left(G_{1}^{2}-F_{1}^{2}\right)=G_{2} F_{2}\left(G_{2}^{2}-F_{2}^{2}\right)=G_{3} F_{3}\left(G_{3}^{2}-F_{3}^{2}\right)
$$

Any solution of this system of equations produces a triple of Heron triangles of $\left(G_{i}^{2}+F_{i}^{2}\right.$, $\left.G_{i}^{2}+F_{i}^{2}, 4 G_{i} F_{i}\right)$ with two equal sides and equal areas.

Take the three sets of generators $G_{1}=r^{2}+r s+s^{2}, F_{1}=r^{2}-s^{2}, G_{2}=r^{2}+r s+s^{2}$, $F_{2}=2 r s+s^{2}, G_{3}=r^{2}+2 r s, F_{3}=r^{2}+r s+s^{2}$.

Then, the right triangle generated by each triple $\left(G_{i}^{2}-F_{i}^{2}, 2 G_{i} F_{i}, G_{i}^{2}+F_{i}^{2}\right)$ has common area $\triangle=r s(2 r+s)(r+2 s)(r+s)(r-s)\left(r^{2}+r s+s^{2}\right)($ see [6]).

As an example, by taking $r=2, s=1$, we get the three triangles $(58,58,84),(73,73,140)$ and $(113,113,224)$ with the areas equal to 1680.

\section{Acknowledgements}

We are grateful to the anonymous referees for careful reading of the paper and giving several useful comments which improved the quality of this paper. 


\section{References}

[1] Adler, A., \& Cloury, J. E. (1995). The Theory of Numbers: A Text and Source Book of Problems. Boston.: Jones and Bartlett Publishers.

[2] Alter, R., \& Curtz, T. B. (1974). A note on congruent numbers. Math. Comp. I, 303-305.

[3] Alter, R., \& Curtz, T. B., \& Kubota, K. K. (1972). Remarks and results on congruent numbers. Proc. Third Southeastern Conf. on Combinatorics, Graph Theory and Computing, pp. 27-35.

[4] Basor, E., \& Hart, B. A Trillion Triangles. Available online: http://www . a imath . org/news/congruentnumbers/.

[5] Bastein, L., (1915). Nombers congruents. Intermediaire des Math, 22, 231-232.

[6] Beiler, A. H. (1966). "Chapter 14: The Eternal Triangle." Recreations in the Theory of Numbers: The Queen of Mathematics Entertains. New York: Dover.

[7] Birch, B. J. (1968). Diophantine analysis and modular functions, Proc., Bombay Colloq. Alg. Geom., 76, 35-42.

[8] Birch, B. J. (1970). Elliptic curves and modular functions, Symp. Math. 1st. Alta MAt., 4, 27-32.

[9] Carlson, J. R. (1970). Determination of Heronian triangles, Fibonacci Quarterly, 8, 499506.

[10] Dickson, L. E. (1934). History of the Theory of Numbers, Vol. II: Diophantine Analysis, G. E. Stechert Co., New York.

[11] Elkies, N. (1988). On $A^{4}+B^{4}+C^{4}=D^{4}$. Mathematics of Computation, 51 (184), 825-835.

[12] Feng, K. (1996). Non-congruent numbers, odd graphs and the Birch-Swinnerton-Dyer conjecture, Acta Arith., 75, 71-83.

[13] Gerardin, A. (1915). Nombers congruent. Intermediaire des Math., 22, 52-53.

[14] Gross, B. H., \& Zagier, D. B. (1986). Heegner points and derivatives of L-series, Invent. Math, 84 (2), 225-320.

[15] Heegner, K. (1952). Diophantine analysis und Modulfunctionen, Math. Z., 56, 227-253.

[16] Izadi, F. (2015). Coungruent number via the pell equation and its analogous counterpart, Notes on Number Theory and Discrete Mathematics, 21 (1), 70-78.

[17] Lagrange, J. (1974-1975). Nombres congruents et courbes elliptiques. Sm. Delange-PisotPoitou (Thorie des nombres), 16e anne, no. 16, 17 p. 
[18] Lagrange, J., Construction dune table de nombres congruents. Manuscript, Reims.

[19] Lander L. J. \& Parkin, T. R. (1966). Counterexamples to Euler's conjecture on sums of like powers, Bull. Amer. Math. Soc., 72, p. 1079.

[20] Monsky, P. (1990). Mock Heegner points and congruent numbers, Math. Z., 204, 45-68.

[21] Nagell, T. (1929). Lanalyse indetermineede degre superieur. Gauthier-Villars, Paris, 39, $16-17$.

[22] Nagell, T. (1981). Introduction to Number Theory, New York, Chelsea Publishing Company.

[23] Piezas III, T. (2010). A Collection of Algebraic Identities, Available online: https:// sites.google.com/site/tpiezas/Home.

[24] Sastryt, K. R. S. (2001). A Heron difference, Crux Math. \& Math. Mayhem, 27, 22-26.

[25] Stephens, N. M. (1975). Congruence properties of congruent numbers, Bull. London Math. Soc., 7, 182-184.

[26] Tunnell, J. B. (1983). A classical Diophantine problem and modular forms of weight 3/2. Inv. Math.. 72 (2), 323-334.

[27] Washington, L. C. (2008). Elliptic Curves: Number Theory and Cryptography, ChapmanHall.

[28] Yiu, P. (1998). Isosceles triangles equal in perimeter and area, Missouri J. Math. Sci., 10, 106-111.

[29] Yiu, P. (1998). Construction of indecomposable Heronian triangles, Rocky Mountain J. of Math., 28, 1189-1201. 